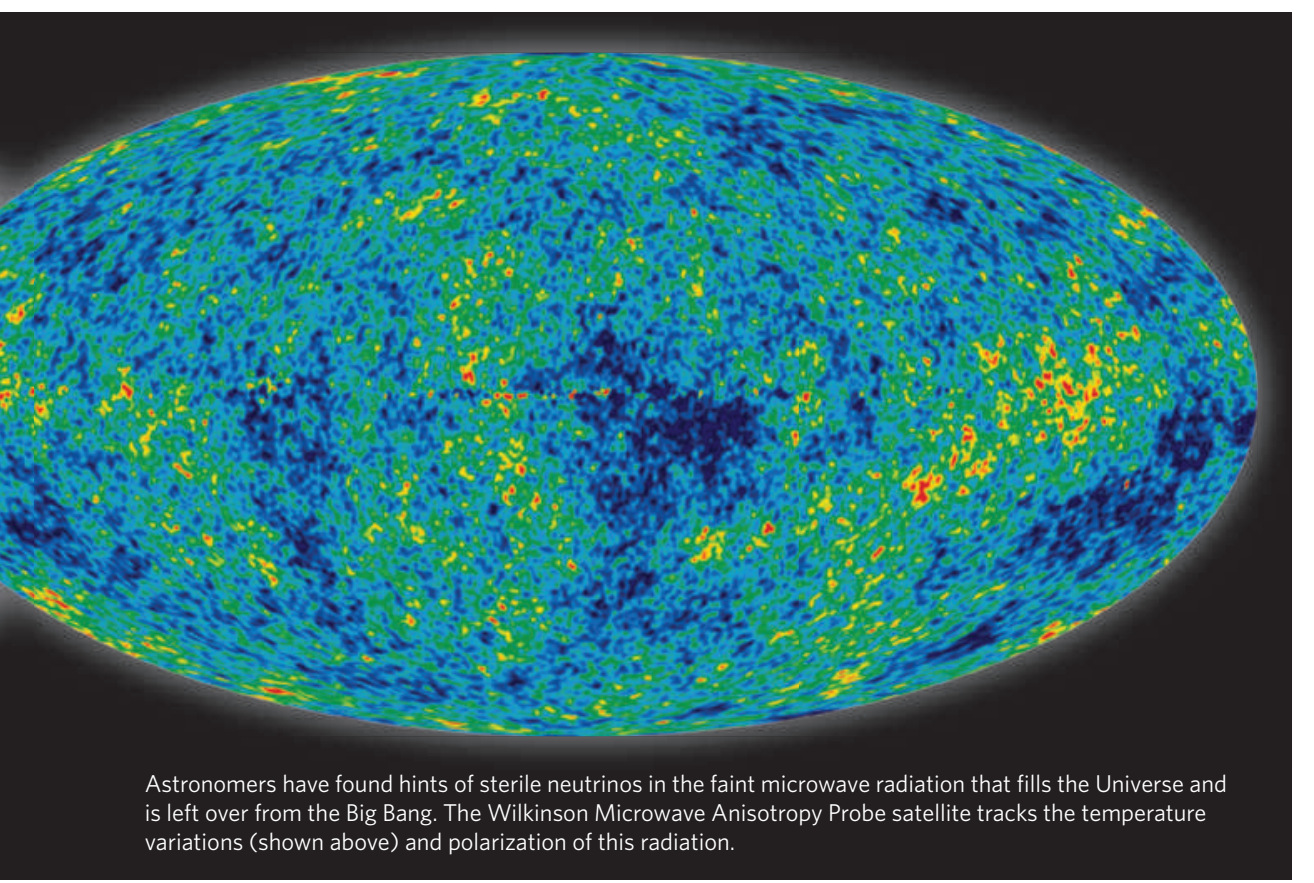

explosions cause neutrons to fuse together, creating elements heavier than iron that end up in other stars and in planets. But they also produce inordinate numbers of neutrinos, which should, in theory, inhibit fusion because they kill off neutrons by changing them into protons and electrons.

Sterile neutrinos could provide a way out. If some of the neutrinos created in supernovae morphed into sterile ones, they would fly off without interacting with neutrons, leaving many of them intact. "We're very interested in this, because the stakes are so high," says Fuller.

\section{Mixed messages}

Sobering news for the sterile-
"The question of sterile neutrinos is absolutely crucial for nuclear particle physics and astrophysics." at Los Alamos in which Louis took part. From 1993 to 1998 , he and his colleagues used the Liquid Scintillator Neutrino Detector experiment to shoot antineutrinos - neutrinos' antimatter counterparts - into 167 tonnes of mineral oil. Their data on how antineutrinos switch from one species to another suggested four distinct flavours. A decade later, data from a follow-up test also at Fermilab - the Mini Booster Neutrino Experiment, or MiniBooNE - found no evidence of a fourth neutrino type. But the MiniBooNE had used a beam of neutrinos, making it difficult to compare its results with the antineutrino data from Los Alamos. Now, after collecting data for a year and a half with antineutrinos, the MiniBooNE experiment sees a different pattern (see 'Neutrino hunters'). When combined with other antineutrino experiments worldwide, says Louis, its data fit "beautifully" to a $3+1$ model: three ordinary neutrinos plus a sterile one ${ }^{4}$.

Louis, who is also a part of the MiniBooNE collaboration, says that the next batch of data, better idea of whether this fleeting interloper has finally been caught.

\section{Eric Hand}

\section{Komatsu, E et al. Preprint at http://arxiv.org/ abs/1001.4538v1 (2010)}

2. Lowenstein, M. \& Kusenko, A. Preprint at http://arxiv. org/abs/0912.0552 (2009).

3. Adamson, P. et al. Phys. Rev. D 81, 052004 (2010). Sorel, M. Phys. Rev. D 80, 073001 (2009). to be released this summer, should give a

4. Karagiorgi, G., Djurcic, Z., Conrad, J. M., Shaevitz, M. H. \&

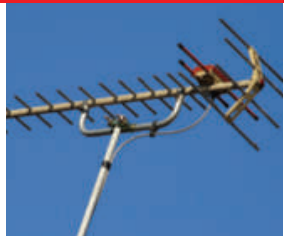

GUIDING LIGHT IN QUANTUM NETWORKS Tiny antennas could direct photons on a chip.

go.nature.com/ruNCbv

\section{Hobbit origins pushed back}

When the remains of tiny hominins nicknamed hobbits - were found on the isolated Indonesian island of Flores in 2003, it sparked an epic hunt to understand the origins of these diminutive cousins of modern humans.

Now, discoveries of stone flakes used as primitive tools on the island suggest that the hobbit's ancestors were there a million years ago, at least 120,000 years earlier than previously thought (A. Brumm et al. Nature doi:10.1038/nature08844; 2010). "Whatever species made it to the island 1 million years ago, it was probably an ancestor of Homo floresiensis," says William Jungers, an anthropologist at Stony Brook University in New York.

The metre-high $H$. floresiensis lived on the island until at least 17,000 years ago, and its small stature probably evolved in response to the island's sparse resources. The simple stone tools demonstrate the skills of its ancestors - people who must have hopscotched across islands from mainland Asia, traversing deep and swift ocean channels, before arriving on Flores.

In 2005, Adam Brumm, an archaeologist at the University of Wollongong in Australia, found the first of about 45 stone tools while exploring a bowl-shaped gully on the island that was like "a hot, steamy wok". Three years later, researchers at Roskilde University in Denmark analysed the ratio of two isotopes of argon trapped in volcanic ash overlaying the tools to determine their age.

Previous tool discoveries showed that hominins had arrived on Flores by 880,000 years ago, suggesting that the hobbit's ancestors might have wiped out some of the island's peculiar indigenous animals, such as the pygmy elephant-like Stegodon sondaari and giant tortoises (Geochelone $s p p$.), which both disappeared at around the same time.

The new finds imply that the hobbit's ancestors coexisted with the creatures for much longer, raising the possibility that a natural disaster was behind the disappearance of the animals.

The team will return to Flores this summer, hoping to find older sediments that could hold earlier evidence of the island's first hominins.

Rex Dalton original evidence came from an experiment 